\title{
Ultrasonication-Assisted Spray Ionization Mass Spectrometry for the Analysis of Biomolecules in Solution
}

\author{
Tsung-Yi Chen, Jia-Yi Lin, Jen-Yi Chen, and Yu-Chie Chen \\ Department of Applied Chemistry, National Chiao Tung University, Hsinchu, Taiwan
}

\begin{abstract}
In this paper, we describe a novel technique-ultrasonication-assisted spray ionization (UASI) - for the generation of singly charged and multiply charged gas-phase ions of biomolecules (e.g., amino acids, peptides, and proteins) from solution; this method employs a low-frequency ultrasonicator (ca. $40 \mathrm{kHz}$ ) in place of the high electric field required for electrospray ionization. When a capillary inlet is immersed into a sample solution within a vial subjected to ultrasonication, the solution is continually directed to the capillary outlet as a result of ultrasonication-assisted capillary action; an ultrasonic spray of the sample solution is emitted at the outlet of the tapered capillary, leading to the ready generation of gas-phase ions. Using an ion trap mass spectrometer, we found that singly charged amino acid and multiply charged peptides/proteins ions were generated through this single-step operation, which is both straightforward and extremely simple to perform. The setup is uncomplicated: only a low-frequency ultrasonicator and a tapered capillary are required to perform UASI. The mass spectra of the multiply charged peptides and proteins obtained from sample solutions subjected to UASI resemble those observed in ESI mass spectra. (J Am Soc Mass Spectrom 2010, 21, 1547-1553) (C) 2010 American Society for Mass Spectrometry
\end{abstract}

$\mathrm{M}$ atrix-assisted laser desorption/ionization mass spectrometry (MALDI MS) [1] is used routinely in biochemical analysis. With the recent rapid growth in nanotechnology, several inorganic nanomaterial-assisted laser desorption/ionization processes [2-5] such as surface-assisted laser desorption/ ionization (SALDI) [3, 4] and desorption/ionization on porous silicon (DIOS) [5] have been attracting attention. Using these techniques, intense laser irradiation is generally required to provide sufficient energy to direct analytes from the condensed phase to the gas phase. Notably, the development of desorption electrospray ionization (DESI) [6] - using a fine spray of charged droplets as the desorption/ionization source for analytes under ambient conditions-has opened up new avenues for progress in desorption/ionization mass spectrometry. Apparently, as long as analyte molecules are provided with sufficient energy, which is not limited to the use of lasers, gas-phase ions can be readily generated from condensed phase samples. Furthermore, this discovery has also provided a fillip to the development of ambient mass spectrometry [7-11]. For example, high-energy laser [8], heated gas jets [9], low-temperature plasma [10], and nitrogen gas [11] were successfully employed to desorb analytes at am-

Address reprint requests to Dr. Y.-C. Chen, Department of Applied Chemistry, National Chiao Tung University, 1001 Ta Hsueh Road, Hsinchu 300, Taiwan. E-mail: yuchie@mail.nctu.edu.tw bient condition. However, post-ionization was generally required for these techniques.

Ultrasonically assisted electrospray ionization [12, 13] was first demonstrated more than a decade ago, using an ultrasonic transducer to enhance the nebulization efficiency of analytes eluted from a liquid chromatography column and then subjecting them to ESI. A method, so called sonic spray ionization (SSI), for small organics and drugs [14-17] has been developed, in which a solution from a fused-silica capillary is sprayed with a sonic gas flow coaxial to the capillary. When analyzing large molecules such as proteins, an electric field is applied to the solution in the capillary to increase the charge density of produced droplets and multiply charged ions of proteins are then generated $[18,19]$. Lately, ultrasonic transducer-based nebulizers (operated at megahertz frequencies or greater) [20, 21] have been employed to introduce desorbed species into an ionization region.

Although these inventions are fascinating, we were interested in developing an alternative approach that eliminates the need for post-ionization. We suspected that low-frequency ultrasonicators, which are generally available in most laboratories, would provide sufficient energy to direct analytes from solution to the gas phase. At first glance, this idea might seem preposterous; from consideration, however, of the operating principles of ultrasonicators, we were motivated to conduct the first ultrasonication-assisted spray ionization (UASI) experiments using samples in solution. Ultrasound is used 
widely at frequencies greater than $20 \mathrm{kHz}$ in chemistry and medical diagnostics because such long wavelengths do not induce chemical changes in molecules in solution-rather, acoustic cavitation occurs. As a result, vapor- and gas-filled voids are formed in the liquid, leading to implosive bubble collapse and associated shock waves [22]. Bubble collapse may result in highspeed jets of droplets and local extremes of temperature and pressure in the liquid [22]. It has been predicted that the internal temperature within these collapsing bubbles can reach several thousand Kelvin [23]; the amount of energy generated should be sufficient to allow phase transitions from the condensed phase to the gas phase and, hence, the transfer of samples in solution to the gas-phase. To prove the concept, a straightforward UASI experiment was conducted and demonstrated in this study.

\section{Experimental}

\section{Reagents and Materials}

Amino acids, peptides, and proteins were purchased from Sigma (St. Louis, MO, USA). Methanol and hydrofluoric acid were obtained from Tedia (Fairfield, $\mathrm{OH}$, USA); acetonitrile, acetic acid, and ammonium hydrogen carbonate were obtained from Riedel de Haën (Seelze, Germany). The fused-silica capillary (50- $\mu \mathrm{m}$ i.d. $\times 365-\mu \mathrm{m}$ o.d.) was obtained from Polymicro Technologies (Phoenix, AZ, USA).

\section{Fabrication of the Tapered Capillary}

Tapered capillaries were fabricated based on the method we described previously [24-26]. Briefly, the tapered fused-silica capillary was fabricated by applying a small weight $(50 \mathrm{~g})$ on the lower end of a vertical capillary. The lower part of the capillary was heated and then quickly drawn to form a narrow capillary tip. After cooling to ambient temperature, the capillary tip was immersed in an HF solution (24\%) for $10 \mathrm{~min}$. The tip was rinsed with methanol and deionized water. The tapered capillary was conditioned before analysis by using a pump (pressure: $18 \mathrm{~mm} \mathrm{Hg}$ ) to rinse it successively with $1 \mathrm{~N} \mathrm{NaOH}$ (30 min), water (30 min), and running buffer (10 $\mathrm{min})$.

\section{Setup of UASI MS}

UASI experiments were performed using a powertunable $(360 \sim 600 \mathrm{~W})$ ultrasonicator $(50 \mathrm{~cm} \times 30 \mathrm{~cm} \times$ $20 \mathrm{~cm}$, frequency: $40 \mathrm{kHz}$ ) (Taipei, Taiwan), generally filled with $8 \mathrm{~L}$ of water. After rinse and activation, the tapered capillary (length, $40 \mathrm{~cm}$; tip diameter: ca. $10 \pm$ $3 \mu \mathrm{m})$ was filled with sample solution before placing into the aqueous sample solution within a vial that was positioned in an ultrasonicator (see Scheme 1). The tapered capillary outlet was close to (ca. $5 \mathrm{~mm}$ ) the inlet of an ion trap mass spectrometer. The heated transfer

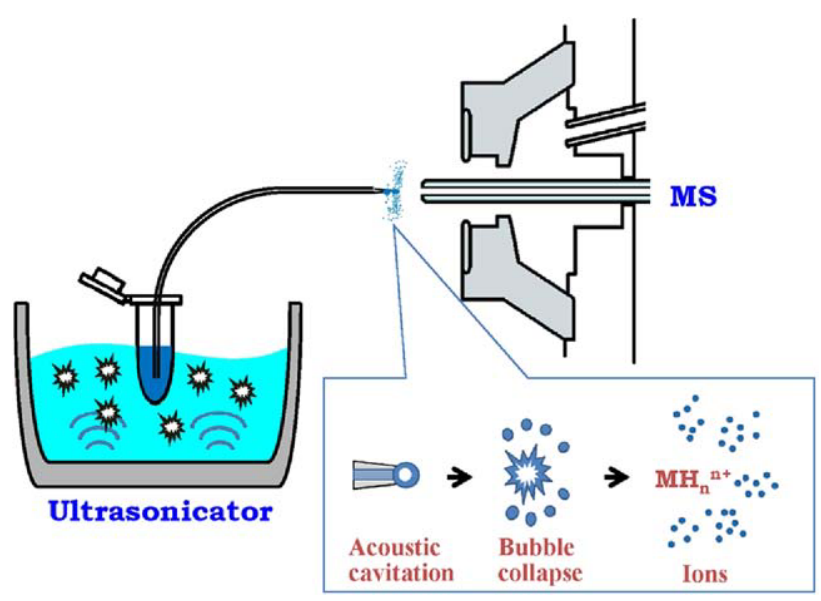

Scheme 1. Cartoon representation of the UASI MS setup and the proposed process for directing analytes from solution to the gas phase.

glass-capillary in the ion trap mass spectrometer was maintained at $150{ }^{\circ} \mathrm{C}$ with a dry gas flow of $5 \mathrm{~L} / \mathrm{min}$. The voltages on the MS capillary inlet were set at -1500 and $+1500 \mathrm{~V}$ for the spectra recorded in positive and negative ion modes, respectively. The on-line nanospray mode was employed during UASI MS analysis. The flow rate was also estimated, i.e., $\sim 0.15 \mu \mathrm{L} / \mathrm{min}$, by using deionized water/acetonitrile $(1: 1, \mathrm{vol} / \mathrm{vol})$ as the running solvent and bradykinin as the sample. When the power of the ultrasonicator was switched on, UASI signals were readily acquired by the ion trap mass spectrometer.

\section{Instrumentation}

All mass spectra were obtained using an Esquire 2000 ion trap mass spectrometer (Bruker Daltonics, Bremen, Germany). When the commercial spray emitter was used for ESI, the spray voltage applied on the MS capillary inlet was set at $-4 \mathrm{kV}$, the flow rate for sample infusion was set at $4 \mu \mathrm{L} / \mathrm{min}$, the pressure of the nebulizer gas was set at $10 \mathrm{psi}$, and the temperature of the heated transfer capillary was maintained at $300{ }^{\circ} \mathrm{C}$ with a dry gas flow $(5 \mathrm{~L} / \mathrm{min})$.

\section{Results and Discussion}

To demonstrate the feasibility of using the UASI setup for obtaining gas-phase ions from solution, we used an amino acid mixture as the sample for examination. Figure 1 displays the UASI mass spectrum of the mixture containing valine, phenylalanine, and tryptophan. The protonated pseudomolecular ions of valine, phenylalanine, and tryptophan at $m / z$ 118.3, 166.2, and 205.1, respectively, appear in the mass spectrum. The inset displays the corresponding plot of the total ion current (TIC) monitoring. The ions can be generated continuously. The results indicate this UASI approach is capable of generating gaseous ions of polar organics 


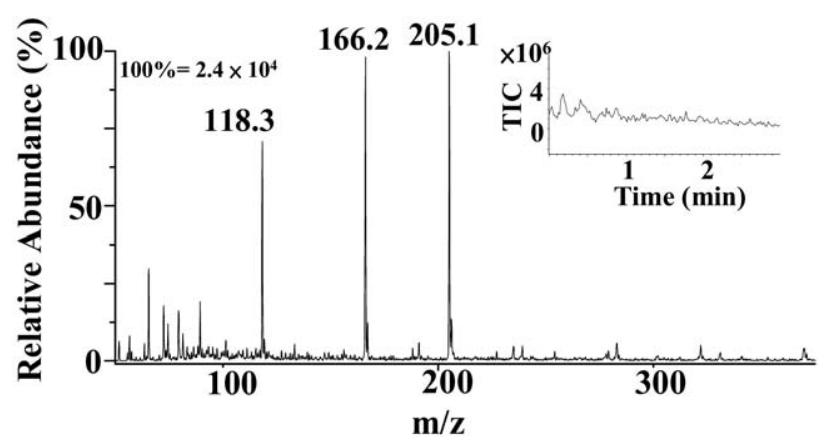

Figure 1. UASI mass spectrum of the amino acid mixture including valine $\left(10^{-6} \mathrm{M}\right)$, phenylalanine $\left(10^{-6} \mathrm{M}\right)$, and tryptophan $\left(10^{-6}\right.$ $\mathrm{M})$ prepared in deionized water. The inset is the corresponding plot of TIC monitoring.

like amino acids directly from solutions. Previously, we demonstrated that a pulled bare fused-silica capillariesnot subjected to an applied external high voltage-can be used to interface capillary electrophoresis with mass spectrometry [23]. Note that we did not treat the surface of the tapered capillary outlet with any electrically conductive materials in this UASI MS approach.

Since the above results have demonstrated that polar amino acids can be analyzed using the UASI MS approach, we were curious whether the UASI MS approach was suitable for the analysis of peptides. Furthermore, to closely examine the details of the UASI process, we monitored the capillary outlet during UASI operation by video (see Supplementary Materials, which can be found in the electronic version of this article). Figure 2a displays the corresponding plot of the total ion monitoring (TIC) when aqueous bradykinin was used as the sample and the selected ion monitoring (SIM) at $m / z 531$, i.e., doubly charged ion of bradykinin. The power of the ultrasonicator was switched on at the time of $34 \mathrm{~s}$ and off at $4 \mathrm{~min} 3 \mathrm{~s}$. The bradykinin signals appeared during the UASI operation and quickly disappeared after the ultrasonicator was switched off. Figure $2 \mathrm{~b}$ displays the corresponding mass spectrum during the UASI operation. The doubly charged ion of bradykinin at $m / z 530.8$ dominates the mass spectrum. The mass peak pattern was similar to what we obtained when using conventional ESI for the analysis of the same sample (Figure 2c). The ion counts obtained from UASI is lower than that obtained from conventional ESI. The flow rate when operating conventional ESI was $4 \mu \mathrm{L} / \mathrm{min}$. However, the UASI spray was operated by nanospray mode with a flow rate of $\sim 0.15 \mu \mathrm{L} / \mathrm{min}$. As a result, fewer ions were generated from UASI, leading to lower ion counts. Nevertheless, the signal-to-noise $(\mathrm{S} / \mathrm{N})$ for the peak at $m / z 530.8$ obtained by UASI MS is better than that obtained from ESI MS (see Figure $2 b$ and c). The results implied that our UASI-MS approach can obtain better ionization efficiency and lead to better spectral quality. It may be contributed from the ionization mechanism of UASI although its mechanism is not clear yet in this initial study. When the ultrasonicator was switched on (after $34 \mathrm{~s}$ ), the capillary tip vibrated noticeably, arising from the action of power-on. However, it quickly returned to a steady-state within seconds as the visible bubbles came out from the outlet. Apparently, bubbles are readily formed from the outlet via acoustic cavitation. Afterwards, a small ring of liquid hangs around the capillary outlet with noticeable vibration.

Figure 3 shows the photographs taken from the supplementary video. Panel a displays the photograph taken before the ultrasonicator was switched on, while panel $\mathrm{b}$ displays the photograph taken after the ultrasonicator was switched on. During the vibration, gas ions seemed readily generated from the ring since singly- and multiply-charged ions were then therefore monitored by the mass spectrometer during the process as we observed in the resultant UASI mass spectra. After the ultrasonicator was switched off (at $4 \mathrm{~min} 3 \mathrm{~s}$ ), the ring of the liquid on the capillary tip suddenly disappeared and the analyte ions faded away simultaneously. As mentioned earlier, it has been known that ultrasonication can cause acoustic cavitation, leading the formation of the vapor- and gas-filled voids in the
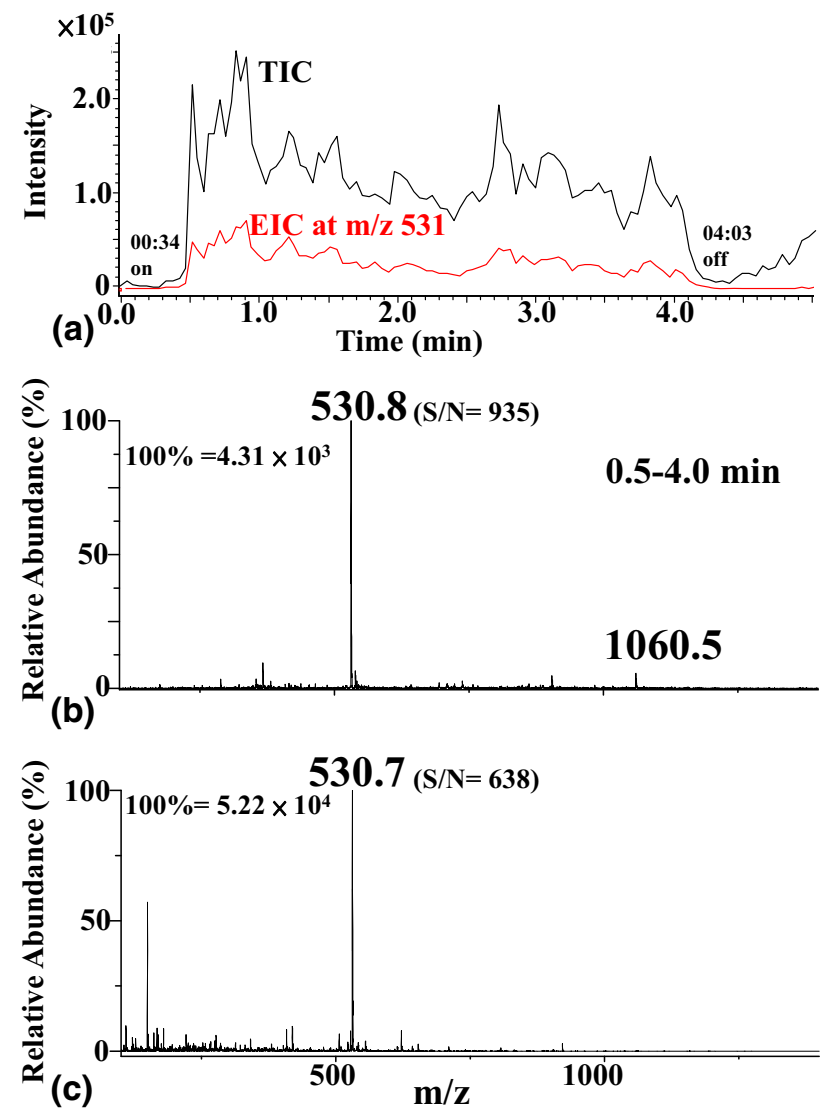

Figure 2. (a) Plots of the total ion current (TIC) and selected ion monitoring (SIM) at $\mathrm{m} / \mathrm{z} 531$ for the supplementary video film. Aqueous bradykinin $\left(10^{-6} \mathrm{M}\right)$ was used as the sample. The ultrasonicator was switched on/off at the time of 00: 34 and 04:03, respectively. (b) Corresponding mass spectrum during the UASI operation (0.5-4 min). (c) ESI mass spectrum of aqueous bradyki$\operatorname{nin}\left(10^{-6} \mathrm{M}\right) . \mathrm{S} / \mathrm{N}=$ signal to noise ratio. 


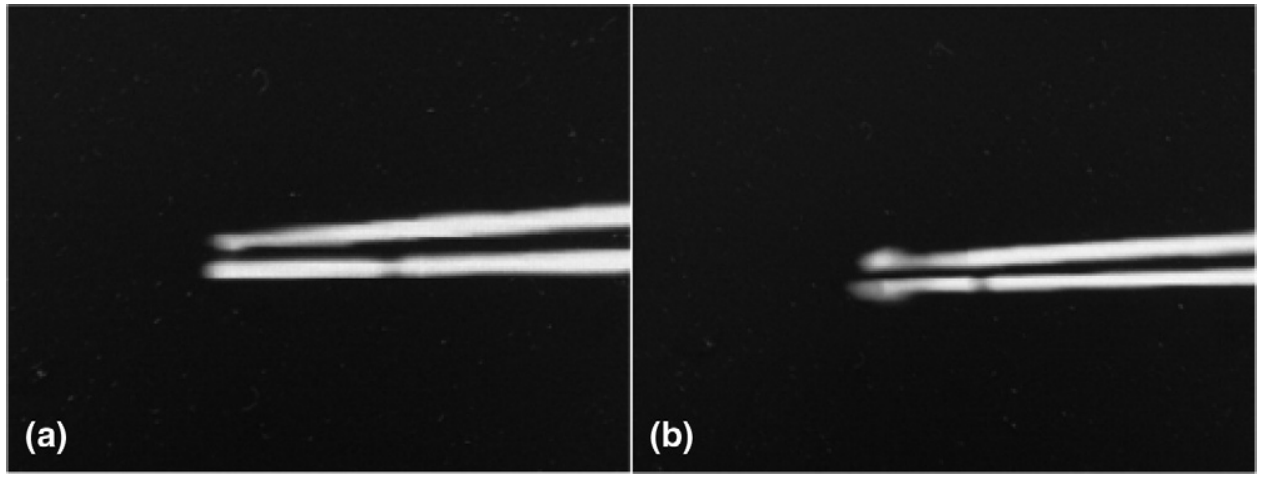

Figure 3. Photographs were taken from the supplementary video: (a) before and (b) after the ultrasonicator was switched on.

liquid. This phenomenon coincides with our observation herein. It has been estimated that the internal temperature within these collapsing bubbles can reach several thousand Kelvin and therefore generate plentiful energy [22], which allows the possibility of the phase transitions of samples from the condensed phase to take place. In the UASI experiment, initial formation of bubbles occurs during the development of the ultrasonic spray from the tapered capillary outlet. After the bubbles collapse, a nanometer-sized liquid jet is formed, subsequently resulting in gas ions after solvent evaporation. Accumulation of multiple charges occurs in the fine droplets during this process, thereby resulting in the signals of multiply charged protein ions dominating the UASI mass spectra (Scheme 1).

We were also curious to test whether negatively charged bradykinin ions could also be observed using this approach. To our delight, the signal for deprotonated bradykinin $\left(10^{-6}\right)$ appeared at $\mathrm{m} / z$ 1058.4 in the mass spectrum (see Figure S1a) with an S/N ratio of 332. Similarly, only this singly charged bradykinin ion appears in the corresponding ESI mass spectrum obtained in the negative ion mode. Thus, ESI-like mass spectra are obtained when using UASI mass spectrometry. Furthermore, on the basis of our experimental results, gaseous analyte ions can be readily generated using this UASI approach either from aqueous solutions or from water/organic solvent. Additionally, the lowest concentration using bradykinin as the sample we obtained so far using this UASI MS approach is 5 $\mathrm{nM}$. Figure S1b shows the corresponding UASI mass spectrum. The doubly charged ions of bradykinin at $\mathrm{m} / \mathrm{z}$ 530.9 with an S/N ratio of 92 dominate the mass spectrum. That is, the detection limit for bradykinin may be lower to less than $1 \mathrm{nM}$ in terms of a $\mathrm{S} / \mathrm{N}$ ratio of 10 . The ions can be continually obtained during the UASI operation although the TIC plot is not as stable as that obtained in conventional ESI MS. Additionally, although we always conditioned the capillary before UASI experiments, we noted UASI mass spectra could be readily obtained from an unconditioned capillary without flushing with aqueous sodium hydroxide before experiments. The step for conditioning capillary is essential for generation of electro-osmotic flow in capillary electrophoresis, but the driving force herein is ultrasonication. Thus, the condition step taken herein is only for the purpose of rinse to remove undesirable impurities.

Next, we tested the ability of UASI mass spectrometry to examine the proteins, including insulin, ubiquitin, cytochrome $c$, and myoglobin in aqueous solution containing $0.5 \%$ acetic acid (Figure $4 a-d$; corresponding TIC plots are presented as insets to each mass spectrum). In each case, we observed multiply charged protein ions in the mass spectra. These results suggest that the mechanism of ion formation during UASI may be similar to that of ESI, even though no extra electric field is present at the tapered capillary outlet. The capillary tip acts like an ion generator during UASI operation. Ions were readily generated outside of the tip during the bubble collapse followed by solvent evaporation as observed and stated earlier. Thus, a floating potential [27] was presumably generated outside of the capillary tip during UASI operation. Thus, there is still a voltage difference between the capillary outlet and the inlet of the mass spectrometer although we are unable to estimate the floating potential currently. As a result, charge accumulation on the fine droplets also occurs and leads to the generation of ESI-like ions during UASI process.

Although we did not apply any external voltage outside of the UASI capillary outlet, it was interesting to see if there was any difference arising when grounding the UASI capillary. Thus, we put a grounded electrode into the solution of the capillary inlet, varied the spray voltage applied on the MS capillary inlet, and used bradykinin $\left(10^{-5} \mathrm{M}\right)$ prepared in $0.1 \%$ acetic acid/ deionized water/methanol $(1: 1, \mathrm{vol} / \mathrm{vol})$ as the sample for UASI MS analysis. The results showed that the ion counts obtained from the UASI-grounded mass spectrum of bradykinin were higher than that observed in UASI-ungrounded mass spectrum of bradykinin under the same experimental condition (see Figure 5). Furthermore, we observed that the ion intensity for the peak at $\mathrm{m} / \mathrm{z} 530.8$ revealing in the UASI-grounded mass spectra of bradykinin increased 

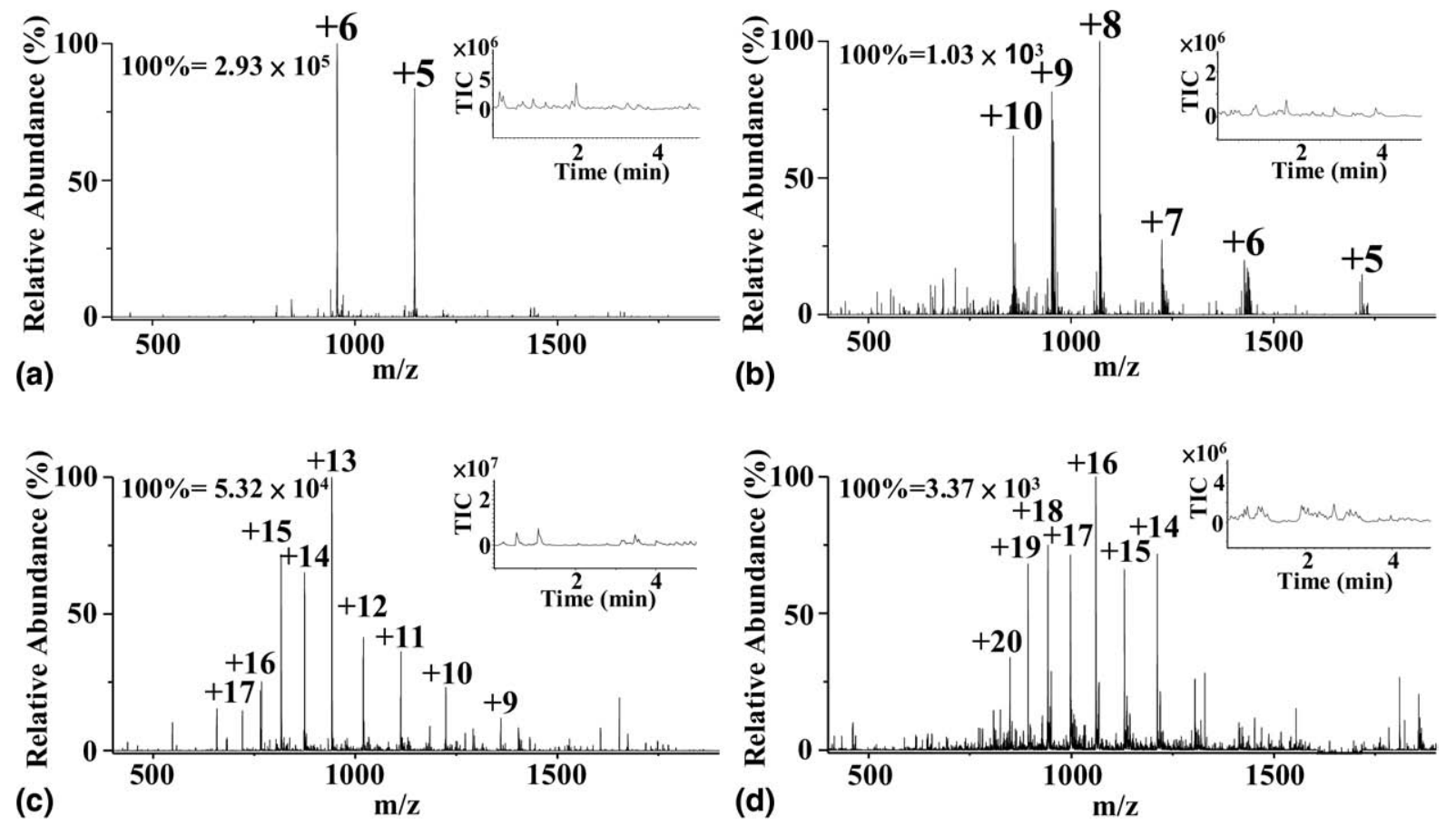

Figure 4. UASI mass spectra of solutions of (a) insulin $\left(10^{-5} \mathrm{M}\right)$, (b) ubiquitin $\left(10^{-5} \mathrm{M}\right),(\mathbf{c})$ cytochrome $c\left(10^{-5} \mathrm{M}\right)$, and $(\mathbf{d})$ myoglobin $\left(10^{-5} \mathrm{M}\right)$. Samples were prepared in deionized water containing $0.5 \%$ acetic acid. Corresponding TIC plots are presented as insets to each mass spectrum.

as the spray voltage set on MS capillary inlet was increased (see Figure S2). However, the ion intensity for the same peak in the UASI-ungrounded mass spectra did not change much when varying the spray voltage. Additionally, the background in the UASIungrounded mass spectrum (Figure $5 \mathrm{~b}$ ) looks much cleaner than that observed in UASI-grounded mass spectrum (Figure 5a).

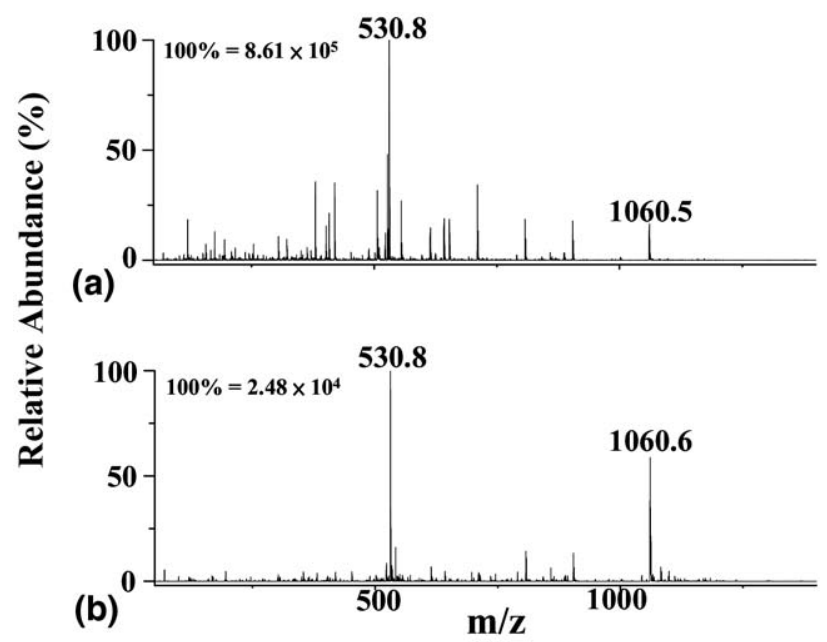

Figure 5. (a) UASI-grounded mass spectrum of bradykinin $\left(10^{-5}\right.$ M) prepared in $0.1 \%$ acetic acid/(deionized water/methanol (1:1, $\mathrm{vol} / \mathrm{vol})$ ). (b) UASI-ungrounded mass spectrum of bradykinin $\left(10^{-5} \mathrm{M}\right)$ prepared in $0.1 \%$ acetic acid/(deionized water/methanol) $(1: 1, \mathrm{vol} / \mathrm{vol})$. The spray voltage set on the MS capillary inlet was $-1500 \mathrm{~V}$.
We also examined the suitability of using the UASI MS approach for obtaining protein ions from solvents with different compositions. Figure 6a displays the UASI mass spectrum of cytochrome $c$ prepared in deionized water/acetonitrile $(1: 1, \mathrm{vol} / \mathrm{vol})$ containing $0.5 \%$ acetic acid. Multiply charged ions of proteins appear in the mass spectrum, which is similar to that obtained from the solvent in the absence of acetonitrile (cf. Figure 4c). Proteins are commonly prepared in buffer solution. Thus, cytochrome $c$ prepared in aqueous ammonium bicarbonate mixed with acetonitrile was used for the examination. Figure $6 b$ present the UASI mass spectrum of cytochrome $c$ prepared in aqueous ammonium bicarbonate $(100 \mathrm{mM})$ /acetonitrile $(1: 1, \mathrm{vol} / \mathrm{vol})$. The mass peak profile is similar to that observed in Figure 4c. Multiply charged ions of cytochrome $c$ are also readily generated from this buffer system by UASI approach. That is to say, the presence of ammonium bicarbonate does not cause unpleasant effect in the performance of UASI approach. When using conventional ESI for the analysis of the same sample, similar results were obtained (see Figure 6c). UASI MS has the same salt tolerance as that conventional ESI does. Additionally, their S/N ratios in both mass spectra are similar. Nevertheless, we noted that the charge distribution of cytochrome $c$ in UASI mass spectrum (Figure 6b) was slightly different from that obtained by ESI MS (Figure 6c). The peak derived from 13-charged cytochrome $c$ ion dominates Figure $6 b$, while the peak derived from 12-charged cytochrome $c$ ion is the base peak in Figure 6c. It seems that the 


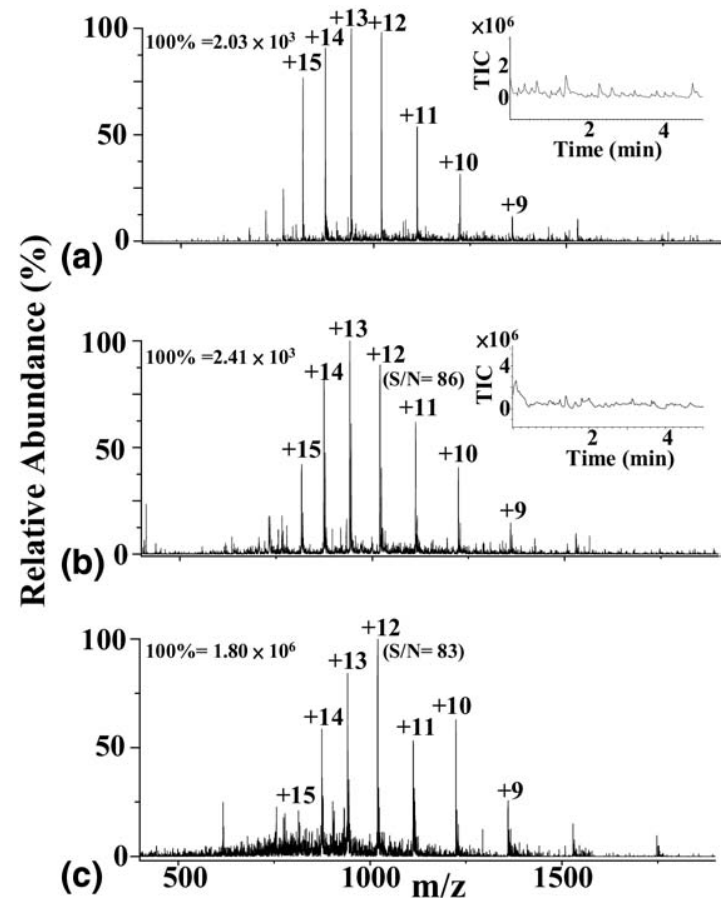

Figure 6. UASI mass spectra of cytochrome $c\left(10^{-5} \mathrm{M}\right)$ prepared in (a) deionized water/acetonitrile ( $\mathrm{vol} / \mathrm{vol}, 1 / 1)$ containing $0.5 \%$ acetic acid and (b) aqueous ammonium bicarbonate $(100 \mathrm{mM}) /$ acetonitrile (vol/vol, 1/1). Corresponding TIC plots are presented as insets to each mass spectrum. (c) ESI mass spectrum of cytochrome $\mathrm{C}\left(10^{-5} \mathrm{M}\right)$ prepared in aqueous ammonium bicarbonate $(100 \mathrm{mM}) /$ acetonitrile $(\mathrm{vol} / \mathrm{vol}, 1 / 1) . \mathrm{S} / \mathrm{N}=$ signal to noise ratio.

structure of cytochrome $c$ unfolded more extensively when conducting UASI than ESI. Presumably, it was contributed from the temperature rising of the UASI sample during ultrasonication. We found that the temperature of the water in the ultrasonicator increase $\sim 1^{\circ} \mathrm{C}$ per $5 \mathrm{~min}$, which may have assisted the unfolding of cytochrome $c$.

On the basis of the results shown above, we believe that the ultrasonication plays two roles in this UASI approach: (1) as the driving force to push the sample solution through the capillary to the outlet; (2) as the energy source to provide sufficient energy for the phase transition of analytes from liquid phase to gas-phase. That is, ultrasonication is in place of the role of a syringe pump in conventional ESI setup and as the driving force to push sample solution through the capillary to the outlet. Furthermore, sufficient energy for phase transition of analytes from condensed phase to gas phase is also simultaneously provided by the ultrasonication based on the experimental results we obtain herein. Ultrasonic spray is also assisted by the sharp capillary outlet because we observed that the analyte signals disappeared when the tip was accidently damaged to a wider hole during the operation. Furthermore, there are no signals observed if the capillary outlet was not fabricated to sharpness. Presumably, the sharp tip is helpful for generation of small droplets. It might be slightly difficult to generate gas ions directly from large liquid droplets in terms of the energy required for phase transition and nebulization efficiency. Additionally, the background in UASI mass spectra seems lower than that obtained in ESI mass spectra.

\section{Conclusions}

We have demonstrated that UASI-MS is suitable for the analysis of biomolecules in this work. The setup of the UASI is extremely simple and the method requires only a single-step operation. We note that because this technique employs ultrasound to provide sufficient energy for the desorption of analytes, the signal is not as stable as that obtained through ESI. Therefore, we do not expect that UASI will provide as good an interface with liquid chromatography as does ESI. Nevertheless, UASI MS provides good spectral quality results with lower background and higher $\mathrm{S} / \mathrm{N}$ ratios compared with ESI MS. Furthermore, UASI MS is capable of being coupled with other interesting techniques. For example, ultrasonicators are used routinely to accelerate reactions and extractions; therefore, we suspect that UASI MS may be appropriate for the on-line monitoring of reactions and extractions performed under ultrasonication-a potentially unique feature of UASI that is not possible with traditional ESI. This work describes only our initial results obtained from the coupling of UASI and MS, and additional studies are required to clarify the actual events occurring during this desorption/ ionization process. We believe that the mass spectral performance will be further improved after more extensive investigation. On this basis, we are very optimistic about the prospects of UASI in chemical and biological mass spectrometry.

\section{Acknowledgments}

The authors thank the National Science Council of Taiwan for financially supporting this research. They are very grateful for helpful discussion with Professor J.-J. Wu (NCKU, Taiwan).

\section{Appendix A Supplementary Material}

Supplementary material associated with this article may be found in the online version at doi:10.1016/ j.jasms.2010.04.021.

\section{References}

1. Karas, M.; Hillenkamp, F. Laser Desorption Ionization of Proteins with Molecular Masses Exceeding 10,000 Daltons. Anal. Chem. 1988, 60, 2299-2301.

2. Tanaka, K.; Waki, H.; Ido, Y.; Akita, S.; Yoshida, T. Protein and Polymer Analyses up to $m / z 100,000$ by Laser Ionization Time-of-Flight Mass Spectrometry. Rapid Commun. Mass Spectrom. 1988, 2, 151-153.

3. Sunner, J.; Dratz, E.; Chen, Y.-C. Graphite Surface-Assisted Laser Desorption/Ionization Time-of-Flight Mass Spectrometry of Peptides and Proteins from Liquid Solutions. Anal. Chem. 1995, 67, 4335-4342.

4. Lo, C.-Y.; Lin, J.-Y.; Chen, W.-Y.; Chen, C.-T.; Chen, Y.-C. SurfaceAssisted Laser Desorption/Ionization Mass Spectrometry on Titania Nanotube Arrays. J. Am. Soc. Mass Spectrom. 2008, 19, 1014-1020. 
5. Wei, J.; Buriak, J. M.; Siuzdak, G. Desorption-Ionization Mass Spectrometry on Porous Silicon. Nature 1999, 399, 243-246.

6. Takats, Z.; Wiseman, J. M.; Gologan, B.; Cooks, R. G. Mass Spectrometry Sampling under Ambient Conditions with Desorption Electrospray Ionization. Science 2004, 306, 471-473.

7. Cody, R. B.; Laramee, J. A.; Durst, H. D. Versatile New Ion Source for the Analysis of Materials in Open Air under Ambient Conditions. Anal. Chem. 2005, 77, 2297-2302.

8. Shiea, J.; Huang, M.-Z.; Shu, H.-J.; Lee, C.-Y.; Yuan, C.-H.; Beech, I.; Sunner, J. Electrospray-Assisted Laser Desorption/Ionization Mass Spectrometry for Direct Ambient Analysis of Solids. Rapid Commun. Mass Spectrom. 2005, 19, 3701-3704.

9. McEwen, C. N.; McKay, R. G.; Larsen, B. S. Analysis of Solids, Liquids, and Biological Tissues Using Solids Probe Introduction at Atmospheric Pressure on Commercial LC/MS Instruments. Anal. Chem. 2005, 77, 7826-7831.

10. Chen, H.; Yang, S.; Wortmann, A. ; Zenobi. R. Neutral Desorption Sampling of Living Objects for Rapid Analysis by Extractive Electrospray Ionization Mass Spectrometry. Angew. Chem. Int. Ed. 2007, 46, 7591-7594.

11. Zhang, X.; Cooks, R. G.; Quyang, Z. Low-Temperature Plasma Probe for Ambient Desorption Ionization. Anal. Chem. 2008, 80, 9097-9104.

12. Banks, J. F.; Shen, S.; Whitehouse, C. M.; Fenn, J. B. Ultrasonically Assisted Electrospray Ionization for LC/MS Determination of Nucleosides from a Transfer RNA Digest. Anal. Chem. 1994, 66, 406-414.

13. Banks, J. F.; Quinn, J. P.; Whitehouse, C. M. LC/ESI-MS Determination of Proteins Using Conventional Liquid Chromatography and Ultrasonically Assisted Electrospray. Anal. Chem. 1994, 66, 3688-3695.

14. Hirabayashi, A.; Sakairi, M.; Koizumi, H. Sonic Spray Ionization Method for Atmospheric Pressure Ionization Mass Spectrometry. Anal. Chem. 1994, 66, 4557-4559.

15. Hirabayashi, A.; Sakairi, M.; Koizumi, H. Sonic Spray Mass Spectrometry. Anal. Chem., 1995, 67, 2878-2882.

16. Dams, R.; Benijts, T.; Gnther, W.; Lambert, W.; De Leenheer, A. Sonic Spray Ionization Technology: Performance Study and Application to A LC/MS Analysis on a Monolithic Silica Column for Heroin Impurity Profiling. Anal. Chem. 2002, 74, 3206-3212.
17. Arinobu, T.; Seno, H.; Ishii, A.; Suzuki, O. Comparison of SSI with APC as an Interface of HPLC-Mass Spectrometry for Analysis of a Drug and Its Metabolites. J. Am. Soc. Mass Spectrom. 2002, 13, 204-208.

18. Hirabayashi, A.; Hirabayashi, Y.; Sakairi, M.; Koizumi, H. MultiplyCharged Ion Formation by Sonic spray. Rapid Commun. Mass Spectrom. 1996, 10, 1703-1705.

19. Hirabayashi, Y.; Hirabayashi, A.; Takada, Y.; Sakairi, M.; Koizumi, H.. A Sonic Spray Interface for the Mass Analysis of Highly Charged Ions from Protein Solutions at High Flow Rates. Anal. Chem. 1998, 70, 1882-1884.

20. Shiea, J.; Chang, D.-Y.; Lin, C.-H.; Jiang, S.-J. Generating Multiply Charged Protein Ions by Ultrasonic Nebulization/Multiple ChannelElectrospray Ionization Mass Spectrometry. Anal. Chem. 2001, 73, 49834987.

21. Zhu, L.; Gamez, G.; Chen, H.; Chingin, K.; Zenobi, R. Rapid Detection of Melamine in Untreated Milk and Wheat Gluten by UltrasoundAssisted Extractive Electrospray Ionization Mass Spectrometry (EESIMS). Chem. Commun. 2009, 5, 559-561.

22. Suslick, K. S.; Flannigan, D. J. Inside a Collapsing Bubble: Sonoluminescence and the Conditions during Cavitation. Annu. Rev. Phys. Chem. 2008, 59, 659-683.

23. Suslick, K. S.; Hammerton, D. A.; Cline, R. E. Sonochemical Hot Spot. J. Am. Chem. Soc. 1986, 108, 5641-5642.

24. Wu, Y.-T.; Chen, Y.-C. Sheathless Capillary Electrophoresis/Electrospray Ionization Mass Spectrometry Using A Pulled Bare Fused-Silica Capillary as the Electrospray Emitter. Anal. Chem. 2005, 77, 2071-2077.

25. Wu, Y.-T.; Chen, Y.-C. Determination of Calcium in Complex Samples Using Functional Magnetic Beads Combined with Electrodeless/ Sheathless Electrospray Ionization Mass Spectrometry. Rapid Commun. Mass Spectrom. 2006, 20, 1995-1999.

26. Hong, C.-Y.; Chen, Y.-C. Selective Enrichment of Ochratoxin A Using Human Serum Albumin Bound Magnetic Beads as the Concentrating Probes for Capillary Electrophoresis/Electrospray Ionization-Mass Spectrometric Analysis. J. Chromatogr. A 2007, 1159, 250-255.

27. Chen, F. F.; Arnush, D. The Floating Potential of Cylindrical Langmuir Probes. Phys. Plasmas 2001, 8, 5051-5052. 